\title{
Automatische Themenextraktion aus gesprochener Sprache
}

\author{
Jürgen Ziegler ${ }^{1}$, Zoulfa El Jerroudi ${ }^{1}$, Carsten Böhm², Wolfgang Beinhauer ${ }^{3}$, \\ Reinhard Busch ${ }^{4}$, Christian Räther ${ }^{5}$ \\ Universität Duisburg-Essen ${ }^{1}$, Universität Leipzig ${ }^{2}$, Fraunhofer Institut (IAO) ${ }^{3}$, linguatec \\ $\mathrm{GmbH}^{4}$, ISA Informationssysteme $\mathrm{GmbH}^{5}$
}

\section{Zusammenfassung}

Bei vielen Formen der Kommunikation und Kooperation in Gruppensitzungen kann das Bereitstellen eines expliziten semantischen Kontextes wertvolle Unterstützung bieten. Semantische Kontexte können das gemeinsame Verständnis eines Problembereichs verbessern, die assoziative Ideenfindung unterstützen, eine moderierende Funktion in der Kommunikation übernehmen oder als Basis für Assistenzfunktionen genutzt werden. In diesem Beitrag wird ein Ansatz präsentiert, in dem semantische Kontexte aus gesprochener Konversation in Echtzeit gewonnen werden. Der vorgestellte Prototyp verknüpft Technologien zur Spracherkennung und zur Analyse unstrukturierter großer Textkorpora miteinander und extrahiert Themenstrukturen aus gesprochener Sprache durch semantisches Matching gegen Terminologiedatenbanken und Ontologien. Die Themenstrukturen werden graphisch visualisiert und in die Diskussion rückgekoppelt, um geeignete Unterstützungsfunktionen anbieten zu können.

\section{$1 \quad$ Einleitung}

Gruppensitzungen und Besprechungen wie etwa Planungsmeetings, Projektstandssitzungen, Entscheidungskonferenzen, Brainstorming Sessions etc. bilden nach wie vor eines der wesentlichsten Instrumente des kooperativen Handelns. Typische Aufgaben in Sitzungen sind etwa die kollaborative Ideenfindung, die Sammlung und Bewertung relevanter Informationen, das Strukturieren erarbeiteter Ideen und Materialien oder der Entwurf gemeinsamer Arbeitsobjekte wie z.B. Dokumente, Projektpläne oder Modellstrukturen.

Eine gemeinsame Eigenschaft kollaborativer Sitzungen, die für alle diese Aufgaben von Bedeutung ist, ist deren Situiertheit, d.h. die Einbettung in einen Beziehungs-, Kommunikations- und Inhaltskontext (Borghoff \& Schlichter 1998), der wesentlich die Handlungsoptionen und -restriktionen der Gruppe beeinflusst. In diesem Beitrag befassen wir uns mit der Frage, wie ein inhaltlicher, semantischer Kontext für Gruppensitzungen erzeugt und auf 
unterschiedliche Weise genutzt werden kann, um insbesondere die kreativen, schwach strukturierten Phasen einer Gruppenaktivität zu unterstützen. Hierzu wird das Konzept der konversationalen Awareness aufgegriffen (Dey et al. 1999), das davon ausgeht, dass durch eine Rückkopplung von Merkmalen des Diskurskontextes in die Gruppe das Kommunikationsverhalten beeinflusst werden kann. Es wird das System TopicMiner vorgestellt, das einen semantischen Kontext automatisch aus der gesprochenen Konversation generiert und durch geeignete Darstellung in die Gruppenaktivität zurückkoppeln kann. Neben einer Förderung der Gruppenwahrnehmung des konversationalen Geschehens auf unterschiedlichen Stufen wird hierdurch auch eine Basis für weiterführende Assistenzfunktionen wie Recherche-, Recommender- oder Strukturierungsfunktionen gelegt.

TopicMiner wurde im Rahmen des Projekts INVITE entwickelt und integriert Methoden der Spracherkennung mit Informationsextraktion und Informationsvisualisierung, um neue Formen der Unterstützung von Gruppenaktivitäten zu explorieren. Die Erstellung dynamischer semantischer Kontexte hat darüber hinaus auch Potentiale in Bereichen wie Information Retrieval, adaptive Benutzungsschnittstellen oder ubiquitäre Systeme.

\section{Szenario}

Der Einsatz des TopicMiners lässt sich mit dem folgenden Szenario veranschaulichen: Produkt- und Marketingexperten eines Finanzdienstleisters treffen sich zu einer BrainstormingSitzung, um Ideen für ein innovatives Finanzprodukt zu entwickeln. Die beteiligten Personen diskutieren in einer Runde mögliche Bedürfnisse im Finanzdienstleistungssektor, um denkbare Produktformen zu identifizieren. Die Teilnehmer der Sitzung sind mit einem kleinen Headset-Mikrophon ausgestattet, über das ihre Äußerungen aufgenommen, durch eine kontinuierliche Spracherkennung erfasst und in einen Textstrom umgewandelt werden. Aus diesem Textstrom werden relevante Begriffe extrahiert und Beziehungen zwischen den Begriffen ermittelt. Die Beteiligten sehen die Themenstruktur ihres Gespräches bereits während ihrer Sitzung als Konzeptnetz (s. Abbildung 1) und können die zusätzlich semantisch assoziierten Begriffe so direkt im Ideenfindungsprozess einbinden. Die Projektion kann innerhalb oder außerhalb des normalen Blickfeldes der Teilnehmer laufen und ggf. nur durch explizite Hinwendung von der Gruppe eingesehen werden, um eine zu starke Fokussierung auf das Konzeptnetz zu vermeiden.

Das Gespräch beginnt mit der Beschreibung der derzeitigen Lage des Aktienmarktes in Deutschland und den potentiellen Auswirkungen der Osterweiterung der EU. Zu den in der Spracheingabe erkannten Begriffen „Deutschland“ und „Osteuropa“ werden stark assoziierte Begriffe wie „Polen“ oder „Slowenien“ angezeigt (s. Abbildung 1). Diese Konzepte werden in der Folge von der Gruppe aufgegriffen, um detailliert die unterschiedlichen Wirtschaftsaussichten in diesen Ländern zu besprechen. Ebenso haben sich ausgehend von weiteren gesprochenen Begriffen wie „Dollar“ oder „Chemieunternehmen“ mehr Strukturen aufgebaut, die den Kontext verdeutlichen und Anstoß für weiterführende Diskussionen sein 
können. Abbildung 1 zeigt einen Ausschnitt aus einem semantischen Netz, das aus einem gestellten Gespräch zwischen zwei Anlageberatern automatisch generiert wurde.

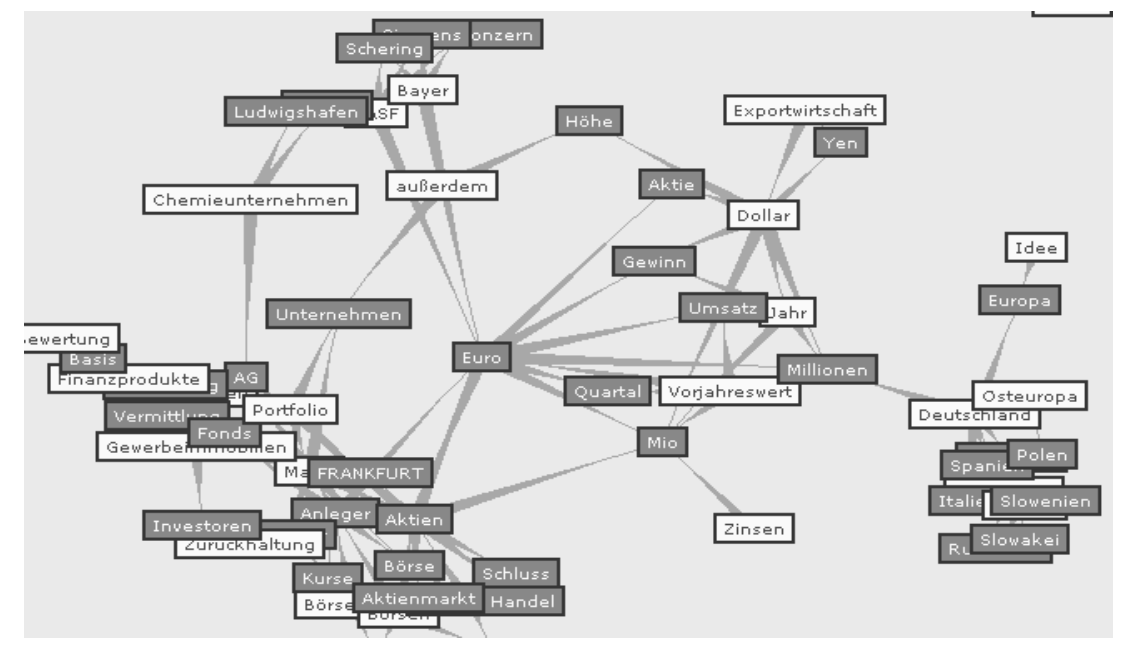

Abbildung 1: Automatisch generiertes semantisches Begriffsnetz

Die Begriffe mit weißem Hintergrund wie „Finanzprodukte“ und „Vorjahreswert“ wurden aus dem Gespräch erkannt. Wörter, die das System als zum semantischen Kontext gehörend erkannt hat, wie „Euro“ und „Umsatz“ werden mit grauem Hintergrund dargestellt. Dieses Netz wächst dynamisch während des Gesprächs, wobei isolierte Begriffe, die keine oder nur schwache Assoziationen zu anderen Konzepten haben, nach einer bestimmten Zeit wieder verschwinden. Weiterhin können durch Analyse des zeitlichen Ablaufs der Diskussion Thementrajektorien auf semantischer Ebene erstellt werden.

Selbstverständlich kann aufgrund des Verwendens und Hintereinanderschaltens mehrerer durch probabilistisches Erkennungsverhalten gekennzeichneter Techniken (kontinuierliche Spracherkennung und auf statistischer Basis gewonnene Themenbeziehungen) kein perfektes Ergebnis erwartet werden. Dennoch liefert der Prototyp bereits ausreichend plausible Ergebnisse, um einen für die Gruppe nutzbaren semantischen Kontext der Diskussion zu erzeugen. Interessanterweise können aber gerade durch den Ansatz, Gesprächsinhalte auf der Ebene semantischer Themenstrukturen zu erkennen, auftretende Fehler in der Spracherkennung kompensiert werden, da Fehlerkennungen aufgrund mangelnder Assoziationen zum bereits aufgebauten Kontext meist nicht zum Tragen kommen. Auf Weiterentwicklungen zur Verbesserung der Extraktionsgüte wird in der Folge noch eingegangen. 


\section{$3 \quad$ Verwandte Arbeiten}

Die Extraktion und Nutzung von aus Sprachströmen gewonnenen Themenstrukturen berührt unterschiedliche Forschungsgebiete wie Spracherkennung, Informationsvisualisierung und CSCW. Zentrale Fragestellungen sind dem Gebiet der Informationsextraktion zuzuordnen. Ziel der Informationsextraktion ist es, domänenspezifische Informationen aus freien Texten aufzuspüren und strukturieren zu können. Überblicke über das Feld der Informationsextraktion geben Cowie und Lehnert (1996) sowie Grishman (1997). Mit dem Thema der Informationsextraktion aus sprachlichen Eingaben, wie Nachrichtensendungen, beschäftigen sich Palmer et al. (1999) und Rigoll (2001). Diese Arbeiten konzentrieren sich darauf, sehr spezifische Informationen wie die Namen von Personen, Organisationen und Orten unter Vorgabe eines Templates zu identifizieren. Das Gebiet der Themenextraktion aus Texten betrachten Bun und Ishizuka (2002). Zur Ermittlung von relevanten Themen werden hier häufigkeitsbasierende Berechnungen für potentiell wichtige Begriffe durchgeführt. Dieser Ansatz lässt sich nur für eine nachträgliche Verarbeitung von Texten einsetzen und ist deshalb nicht echtzeitfähig.

Speziell mit der Themenextraktion aus gesprochener Sprache in Echtzeit beschäftigen sich Jebara et al. (2000), sie verfolgen jedoch einen anderen Ansatz. Während TopicMiner beliebige Themen frei aus dem Gespräch erkennen kann, gibt es in Jebara et al. (2000) eine fest definierte Themenklassifikation von 12 Themen, zu der die erkannten Wörter zugeordnet werden. Die Zuordnung der erkannten Wörter funktioniert aufgrund von Lernverfahren. Das System bekommt zu einem bestimmten Thema eine ganze Anzahl von Dokumenten, die sich mit diesem Thema befassen und „lernt“ so die Wörter, die zu diesem Thema gehören. Fallen zu einem bestimmten Thema eine feste Anzahl an Wörter (z.B. 100), so gilt dieses Thema als erkannt. In ihrem Ansatz berücksichtigen Jebara et al. keine semantischen Kontexte.

Auch DiMicco \& Bender (2004) beschäftigen sich mit Themenextraktion aus gesprochener Sprache. Sie visualisieren Schlüsselbegriffe aus der Gruppendiskussion auf einem gemeinsamen Display, um die Qualität der Gruppenbeiträge durch die Sichtbarmachung verschiedener Gesichtspunkte zu erhöhen. In dieser Arbeit wird jedoch nicht der Versuch unternommen, Beziehungen zwischen diesen Begriffen zu entdecken oder semantisch assoziierte Begriffe abzuleiten, um einen gemeinsamen semantischen Kontext für die Gruppe aufzubauen.

Zur Frage der Unterstützung von Gruppensitzungen durch die Entwicklung gemeinsamer Themenstrukturen existieren bereits seit längerem Ansätze, bei denen das Wissen der Gruppe explizit formuliert und manuell durch visuelle Sprachen modelliert werden muss. Entsprechende Systeme sind u.a. gIBIS (Conklin \& Bergman 1987), Dolphin (Streitz et al. 1998) und DISCBOARD (Gaßner 2003). Diese Systeme erfordern allerdings zusätzlichen Bearbeitungsaufwand und haben noch keine breite praktische Anwendung gefunden (Ausnahmen sind einfache kommerzielle Brainstorming-Systeme wie z.B. MindManager). Sie sind eher auf eine strukturierte, zielgerichtete Erstellung von Diskurs- oder Domänenmodellen ausgerichtet und sind für eine Awareness orientierte, leichtgewichtige Unterstützung des Gruppendiskurses weniger geeignet. 


\section{Architektur und Informationsextraktion}

Das in diesem Beitrag vorgestellte System verknüpft bestehende Technologien zur Spracherkennung und zur Analyse unstrukturierter großer Textkorpora (Text Mining) in neuartiger Weise und erzeugt so eine innovative Anwendung zur Unterstützung der Gesprächsanalyse in gruppenorientierten Sitzungen. Die Architektur des prototypischen Systems gliedert sich dabei in die in Abbildung 2 dargestellten Einheiten.

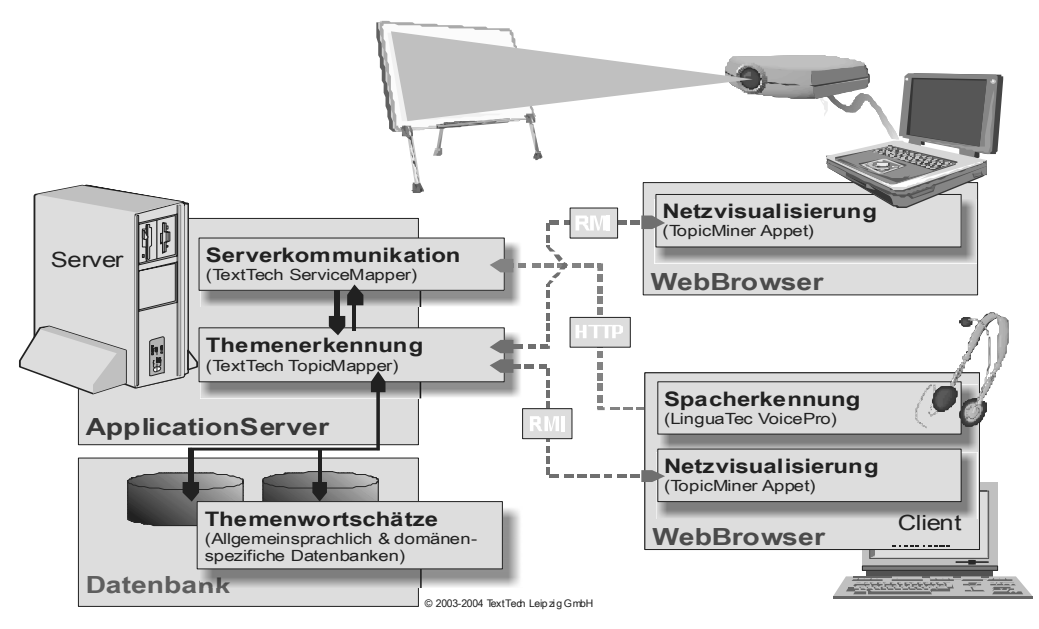

Abbildung 2: Systemarchitektur des TopicMiners

Die Erkennungsmechanismen in TopicMiner sind mehrbenutzerfähig und ermöglichen die simultane Darstellung mehrerer Sprechereingaben in einem kollaborativ entstehenden Begriffsnetzwerk. Für die Erzeugung der Begriffsnetzwerke werden die Spracheingaben der einzelnen Sprecher zunächst durch die Spracherkennungssoftware VoicePro erfasst und in einen Textstrom umgewandelt. Diese Daten gelangen über eine HTTP-Anbindung zu der Serverkomponente des TopicMiners, wo sie zeitlich serialisiert der Themenerkennung zugeleitet werden. Diese bearbeitet die einzelnen im Sprachstrom enthaltenen Wortformen und filtert diejenigen Begriffe und Themen heraus, die eine hinreichende Relevanz für die ausgewählte Domäne besitzen. Die im Gespräch erwähnten Begriffe stellen das Gerüst für das zu erzeugende semantische Netzwerk dar. Wird zu einem erkannten Begriff innerhalb eines bestimmten Zeitfensters kein weiterer assoziierter Begriff erkannt, wird der Begriff wieder verworfen. Weiterhin werden die erkannten Begriffe durch zusätzliche assoziierte Begriffe erweitert, die in die resultierende Begriffsstruktur eingebaut werden. Hierzu werden umfangreiche Netzwerke aus miteinander semantisch assoziierten Begriffen verwendet, die statisch in einer Datenbank abgelegt sind.

Zum Aufbau dieser semantischen Bezüge werden Wortkollokationen gebildet, die folgendermaßen abgeleitet werden. Auf der Basis eines Korpus von etwa 10 Millionen Sätzen un- 
terschiedlichster Quellen (z.B. Zeitungsartikel) wird das signifikant gemeinsame Auftreten zweier Wörter in einem Satz berechnet. Unter Kollokationen verstehen wir Paare von Wörtern, die in einem Bereich (z.B. dem Satz oder direkt benachbart) hinreichend häufig zusammen auftreten. Mit Satzkollokationen werden semantische Bezüge ausgedrückt. Kollokationen können selbst auf großen Korpora automatisch ausgezählt werden. Ihre Bewertung erfolgt aufgrund des Signifikanzwerts, der aus der Anzahl der beteiligten Wörter, der Anzahl des Auftretens der Kollokation und der Korpusgröße berechnet wird. Die daraus resultierenden Graphen spiegeln dann den Kontext wider, in dem das jeweilige Wort auftritt. Für den Filterprozess wird dabei auf eine umfangreiche sprachliche Datenbasis zurückgegriffen, die zum einen allgemeinsprachliche Daten beinhalten (vergleichbar mit der allgemeinen Sprachkompetenz von Muttersprachlern), sowie domänenspezifische Datenbanken, die aus vorhandenen domänenspezifischen Repositories automatisch extrahiert werden können. Sinnvolle Kandidaten für solche fachspezifischen Dokumentenkollektionen sind diejenigen Unterlagen, die für die unterstützte Gruppensitzung relevant sind, möglicherweise ergänzt durch Hintergrundinformationen aus dem firmeninternen Intranet. Obwohl diese Dokumente den Teilnehmern in aller Regel bekannt sein werden, unterstützt die simultane Gesprächsvisualisierung die Gruppenarbeit durch Bezugnahme zu konkreten Konzepten und Zusammenhängen aus den hinterlegten Daten.

Das erzeugte semantische Netz entsteht dabei nahezu in Echtzeit, geringe Verzögerungen ergeben sich durch die kumulierten Laufzeiten bei der Spracherkennung, der Kommunikation der einzelnen Komponenten untereinander und der Filterung der Gesprächsthemen mit den assoziierten Begriffen in dem Netzwerk. Die serverseitigen Komponenten sind dabei für die Filterung der Sprachströme zuständig, die clientseitigen Komponenten sorgen für die dynamische Darstellung der Graphen, insbesondere im Hinblick auf die Anordnung auf dem Bildschirm. Die bidirektionale Kommunikation zwischen Server und Client erfolgt dabei durch Einsatz der Remote-Method-Invocation (RMI). Technisch realisiert ist die Serverkomponente als Web-Applikation, die in einem Servletcontainer ablauffähig ist, die clientseitige Darstellung ist als Java-Applet realisiert.

\section{5 \\ Unterstützung bei der kollaborativen Erstellung von Ontologien}

Ein relevantes Einsatzgebiet für TopicMiner ist die Unterstützung der kollaborativen Erstellung von Themenstrukturen und Ontologien, z.B. für den Aufbau von Metadatenstrukturen für Websites oder Domänenmodellen für bestimmte Wissensbereiche. Die Entwicklung von Ontologien ist eine komplexe Aufgabe, wobei es erste Bestrebungen gibt, diesen Prozess zu strukturieren. Beispielsweise verfolgen Sure et al. (2002) und Holsapple und Joshi (2002) einen kollaborativen Ansatz zur Ontologieentwicklung mit dem Ziel, die Beteiligten möglichst früh in den Entwicklungsprozess zu integrieren, um so die Akzeptanz der Ontologie zu fördern und das Risiko einer lückenhaften Ontologiespezifikation zu verringern. Wesentliche Aufgaben in der ersten Phase der Ontologieentwicklung sind nach Sure et al. (2002) die 
Erstellung einer ersten informalen Taxonomie, die alle relevanten Begriffe aus der Anwendungsdomäne enthält und die durch die Akquisition von Wissen von Domänen-Experten ergänzt wird. Hieraus wird eine erste Basis-Ontologie erstellt, die alle relevanten Konzepte und Relationen beinhaltet. Problematisch ist jedoch in praktischen Anwendungen, dass Anwender typischerweise den für den Prozess der Wissensakquisition erforderlichen Aufwand nicht erbringen wollen.

Hier kann der Einsatz von sprachbasierten Erstellungshilfen den Aufwand für die Wissensakquisition verringern und gleichzeitig ein intuitiveres Mittel für die Erfassung darstellen. TopicMiner bietet insbesondere in der frühen Phase der Ontologieerstellung die Möglichkeit, eine erste Materialsammlung und einen Grobentwurf ohne zusätzlichen Aufwand zu erstellen. So kann z.B. vermieden werden, dass das Gespräch zwischen Entwicklern und Anwendern durch die explizite Nutzung eines Werkzeugs behindert wird. Die mit TopicMiner automatisch generierten Begriffsnetze müssen im weiteren Verlauf des Ontologieentwicklungsprozesses überarbeitet, ergänzt und restrukturiert werden, da die automatische Erkennung realistischerweise keine direkt verwendbaren Strukturen erzeugt. Dazu können sie mit anderen Werkzeugen wie dem im gleichen Projekt entwickelten System MatrixBrowser (Ziegler \& Kunz 2002, s. Abbildung 3) visualisiert und weiterbearbeitet werden. Im System MatrixBrowser werden komplexe Netzstrukturen in Form einer interaktiven Matrix dargestellt und editiert.

Der vorgestellte Ansatz der automatischen Generierung von semantischen Kontexten kann als erster Schritt der kollaborativen Erstellung von ontologischen Strukturen aufgefasst werden. Der semantische Kontext kann dabei prinzipiell aus einer Vielzahl unterschiedlicher Quellen gespeist werden. Hierzu zählen die in der Sitzung selbst erzeugten Materialien, bislang erarbeitete oder verwendete Dokumente, Ergebnisse von Recherchen sowie insbesondere auch die gesprochene Konversation. Die hierfür erforderlichen Mechanismen zur Integration unterschiedlicher, sowohl statischer als auch dynamischer Themenstrukturen sind Gegenstand für zukünftige Forschungsarbeiten.

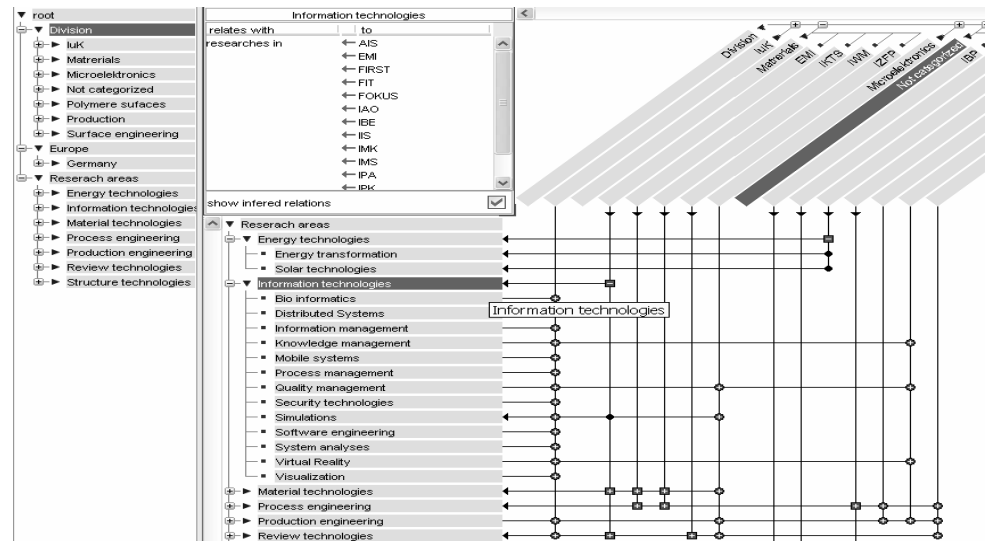

Abbildung 3: Das System Matrix-Browser 


\section{Einsatz- und Anwendungspotentiale}

Durch TopicMiner kann die Kooperation und Koordination von Gruppenarbeit in verschiedenen Situationen unterstützt und beschleunigt werden. Im Folgenden werden verschiedene Fallbeispiele skizziert, in denen TopicMiner Anwendung finden könnte:

- In der Redaktion einer Zeitschrift findet eine Konferenz zu einem aktuellen Thema statt. Da der Gegenstandsbereich neu ist, unterhalten sich die Teilnehmer zunächst über durchzuführende Recherchen. TopicMiner läuft im Hintergrund mit und extrahiert relevante Begriffe. Mit diesen Begriffen wird bereits während des Gesprächs eine Online-Suche in Agenturarchiven und weiteren Quellen angestoßen, die Hintergrundinformationen, Archivbilder oder weitere Begriffe zu dieser Thematik liefert. Die an der Redaktionskonferenz beteiligten Personen können diese Ergebnisse mitverfolgen und hieraus neue Fragen oder Recherchebedürfnisse formulieren, die wiederum vom System ausgewertet werden. Hierdurch kann eine Art von unterstütztem Ideenfindungs-/Recherchezyklus erzeugt werden, der eine raschere und zielgerichtetere Formulierung eines Beitragskonzepts ermöglicht.

- Ein zweites Beispielszenario sind Brainstorming-Sitzungen zur Entwicklung neuer Produkte oder Dienstleistungen. Ein Unternehmen sucht nach innovativen Ideen, um neue Produkte erfolgreich zu entwickeln und zu vermarkten. Dafür muss zunächst ermittelt werden, wo Bedarf bestehen könnte. Die beteiligten Personen erörtern in einer Runde unterschiedliche Ideen. TopicMiner nimmt diese auf, ordnet sie und sucht im Hintergrund nach passenden Informationen. Die Beteiligten sehen diese dann bereits während ihres Gesprächs und können die Informationen so direkt im Ideenfindungsprozess einbinden.

- Auch im medizinischen Kontext ist der Einsatz des TopicMiners denkbar. Der Arzt spricht nach der Untersuchung eines Patienten eine Falldokumentation und eine entsprechende Behandlungsmethode, die er gewählt hat, in das System. Für den Arzt ist dies keine große Umstellung, da er auch bisher schon häufig die Dokumentation und Behandlungsmethode diktiert hat. Mit Hilfe des TopicMiners wäre es möglich, parallel zur Falldokumentation verwandte Fälle oder relevante Fachliteratur zu suchen und bereitzustellen.

\section{7 Bewertung und Ausblick}

Bei dem hier vorgestellten System handelt es sich um einen explorativen Prototypen, der noch keinen Anspruch auf unmittelbare Einsetzbarkeit erhebt. Dennoch zeigt das System TopicMiner erfolgreich einen neuen Ansatz auf, wie durch eine Kombination perzeptiver, auf impliziten Nutzereingaben beruhender Interaktionsformen mit linguistischen und semantischen Methoden sowie Techniken der Informationsvisualisierung neue Unterstützungsszenarien für die Gruppenkooperation ermöglicht werden. Das Konzept der automatisierten Erkennung von semantischen Kontexten hat allerdings auch für weitere Anwendungsfelder 
ein erhebliches Potential, wie z.B. für adaptive Interaktionstechniken, sich selbst konfigurierende Navigationstrukturen oder intelligente Assistenten.

In ersten Demonstrationen und Vorstudien konnte die Realisierbarkeit des Ansatzes gezeigt werden. Hierzu wurden sowohl vordefinierte Gesprächsszenarien als auch freie Diskussionssituationen untersucht. Das Ziel einer Rückkopplung der erkannten Themen mit ausreichendem Echtzeitverhalten wurde erreicht. Die abgeleiteten Begriffsstrukturen wurden sowohl von den Gesprächsteilnehmern als auch von Beobachtern als größtenteils relevant für den tatsächlichen Gesprächsinhalt eingestuft. Die abgeleiteten Begriffe konnten aufgegriffen und in die Diskussion eingebunden werden. Systematischere Evaluationen der Effektivität der Gruppenunterstützung müssen allerdings weiterentwickelten Systemversionen vorbehalten bleiben. Die Spracherkennung ist bislang sprecherabhängig, so dass das System zunächst von den Teilnehmern trainiert werden muss. Gegenwärtig ist nur dadurch eine ausreichende Erkennungsqualität zu erreichen. Allerdings kann dieses Problem für den praktischen Einsatz z.B. durch serverbasierte Sprachprofile gemildert werden. Die Erkennung erwies sich auch bei schnell und beiläufig gesprochenen Texten als überraschend gut. Erkennungsfehler fielen aufgrund des nachfolgenden Matching gegen die terminologische Datenbasis weitaus weniger ins Gewicht, als dies bei einer reinen Diktieranwendung der Fall gewesen wäre. Die Disambiguierung erkennungsbasierter Eingaben anhand des semantischen Kontextes ist deshalb ein viel versprechender Ansatz, der in weiterführenden Arbeiten vertieft wird.

Auf Basis der bisherigen Ergebnisse sollen die Arbeiten in die folgenden Richtungen weitergetrieben werden. Zum einen wird in der Kernkomponente daran gearbeitet, auch typisierte Beziehungen zwischen erkannten Begriffen erstellen zu können. Hierzu werden als Basis typisierte Relationen in der zugrunde liegenden Wortschatzdatenbank erzeugt. Weiterhin soll untersucht werden, wie die dynamische Entwicklung des Gesprächsfokusses besser zu erfassen und darzustellen ist. Hierdurch soll es möglich sein, einen „roten Faden“ der Gesprächsführung zu erkennen und zu dokumentieren. Für die Entwicklung des semantischen Kontextes ist außerdem die Integration zusätzlicher Ontologien oder Themenstrukturen von Bedeutung. Schließlich sollen neue Formen der Visualisierung und Gruppenunterstützung entwickelt werden. Diese sollen auch Möglichkeiten einschließen, die entstehenden Strukturen in der Gruppe interaktiv weiterzuentwickeln und z.B. durch Drag \& Drop-Techniken zu bearbeiten oder miteinander zu verknüpfen.

\section{Literaturverzeichnis}

Borghoff, U.; Schlichter, J. (1998): Rechnergestützte Gruppenarbeit - Eine Einführung in verteilte Anwendungen. Heidelberg: Springer-Verlag.

Bun, M.; Ishizuka, M. (2002): Topic Extraction from News Archive Using TF*PDF Algorithm. In: Ling, T. W.; Dayal, U.; Bertino, E.; Ng, W. K.; Goh, A. (Hrsg.): The Third International Conference on Web Information Systems Engineering (WISE'02). Singapore: IEEE CS Press.

Conklin, J.; Bergman, M. L. (1987): gIBIS: A hypertext tool for team design deliberation. In: Bernstein, M.; Carr, L.; Osterbye, O. (Hrsg.): Proceedings of Hypertext '87 Chapel Hill: ACM Press. S. $247-251$. 
Cowie, J.; Lehnert, W. (1996): Information Extraction. In: Communications of the ACM. Vol. 39, Nr. 1, S. 80-90.

Dey, A. K.; Salber, D.; Abowd, G. D.; Futakawa M. (1999): The Conference Assistant: Combining context-awareness with wearable computing. In: Proceedings of the 3rd International Symposium on Wearable Computers ISWC '99. San Francisco : IEEE Computer Society Press, S. 21-28.

DiMicco, J. M.; Bender, W. (2004): Second Messanger: Increasing the Visibility of Minority Viewpoints with a Face-to-face Collaboration Tool. In: Vanderdonckt, J.; Nunes, N. J.; Rich, C. (Hrsg.): Procceedings of Conference on Intelligent User Interfaces. Funchal, Madeira, Portugal: ACM Press.

Gaßner, K. S. (2003): Diskussionen als Szenario zur Ko-Konstruktion von Wissen mit visuellen Sprachen. Dissertation: Fachbereich Elektrotechnik. Universität Duisburg.

Grishman, R. (1997): Information Extraction: Techniques and Challenges. In: Pazienza, M. T. (Hrsg.): Information Extraction, Lecture Notes in Artificial Intelligence. Rom: Springer-Verlag.

Hernandez, N.; Grau, B. (2003): What Is This Text About? Combining topic and meta- descriptors for text structure presentation. In: Jones, S. B.; Novick, D. G. (Hrsg.): Proceedings of the 21 st annual international conference on Documentation. San Francisco: ACM Press, S. 117-124.

Holsapple, C. W.; Johsi, K. D. (2002): A Collaborative Approch to Ontologie Design. In: Communications of the ACM, 45 (2002), [2], S. 42-47.

Jebara, T.; Ivanov, Y.; Rahimi, A.; Pentland, A. (2000): Tracking conversational context for machine mediation of human discourse. In: Dautenhahn, K. (Hrsg.): AAAI Fall 2000 Symposium - Socially Intelligent Agents - The Human in the Loop. Massachusetts: AAAI Press.

Palmer, D. D.; Burger, J. D.; Ostendorf, M. (1999): Information Extraction From Broadcast News Speech Data. In: Procceedings of DARPA Broadcast News Workshop. S. 41-46.

Rigoll, G. (2001): The ALERT System: Advanced Broadcast Speech Recognition Technology for Selective Dissemination of Multimedia Information. In: Workshop on Automatic Speech Recognition and Understanding - ASRU 2001. Madonna di Campiglio, Italy.

Streitz, N.; Geißler, J.; Haake, J.; Hoi, J. (1994): Dolphin: Integrated meeting support across Liveboards, local and remote desktop environments. In: Frankhauser, P.; Ockenfeld, M. (Hrsg.): Integrated publication and information systems. Darmstadt: GMD IPSI.

Sure, Y.; Erdmann, M.; Angele, J.; Staab, S.; Studer, R.; Wenke, D. (2002): OntoEdit: Collaborative Ontology Development for the Semantic Web. In: Horrocks, I; Hendler, J. A. (Hrsg.): The Semantic Web - ISWC 2002. First International Semantic Web Conference. Sardinia, Italy: Springer Verlag.

Ziegler, J.; Kunz, C. (2002): Matrix Browser - Visualisierung und Exploration vernetzter Informationsräume. In: Herczeg, M.; Prinz, W.; Oberquelle, H. (Hrsg.): Mensch und Computer 2002: Vom interaktiven Werkzeug zu kooperativen Arbeits- und Lernwelten. Stuttgart: B.G. Teubner. 